\section{A126 EVOLUTION OF ECTOPIC LYMPHOID NEOGENESIS AND IN SITU AUTOANTIBODIES PRODUCTION IN AUTOIMMUNE DIABETIC NOD MICE: CELLULAR AND MOLECULAR CHARACTERISATION OF TERTIARY LYMPHOID STRUCTURES IN PANCREATIC ISLETS}

Elisa Astorri, ${ }^{1,2}$ Michele Bombardieri, ${ }^{1}, 2$ Silvia Gabba, ${ }^{1}$ Mark Peakman, ${ }^{3}$ Paolo Pozzilli, ${ }^{4}$ Costantino Pitzalis ${ }^{1}$ Centre for Experimental Medicine \& Rheumatology, William Harvey Research Institute, Barts and The London School of Medicine \& Dentistry, London, UK; ' ${ }^{3}$ Department of Immunobiology, Guy's, King's and St Thomas' School of Medicine, London, UK; ${ }^{4}$ Department of Endocrinology and Diabetes, University Campus Bio-Medico, Rome, Italy

10.1136/ard.2010.148999.2

A pivotal role for tertiary lymphoid structures (TLS) in promoting antigen-specific humoral responses during chronic inflammation is emerging in several autoimmune conditions, including rheumatoid arthritis, Sjogren's syndrome and autoimmune thyroiditis. However, there is limited evidence on the cellular and molecular mechanisms underlying TLS formation and their contribution to autoimmunity in the pancreas during autoimmune insulitis. Here the authors performed a detailed and comprehensive assessment of the evolution of TLS during autoimmune insulitis in 126 female non-obese diabetic (NOD) mice from 4 to 38 weeks of age. The authors demonstrated that during progression from peri-insulitis to intrainsulitis in early diabetic mice, $\mathrm{T}$ and $\mathrm{B}$ cell infiltration follows a highly regulated process with the formation of lymphoid aggregates characterised by $\mathrm{T} / \mathrm{B}$ cell segregation, follicular dendritic cells networks and differentiation of germinal centre $B$ cells. This process is preceded by local upregulation of lymphotoxins $(\mathrm{Lt}) \alpha / \beta$ and lymphoid chemokines (CKs) CXCL13 and CCL19 and is associated with infiltration of B220/IgD+/CD23/CD21 follicular B cells expressing CXCR5. Despite a similar incidence of insulitis, late diabetic mice displayed a significantly reduced incidence of fully organised TLS and reduced levels of Lt/CKs. Upon development, TLS were fully functional in supporting in situ autoreactive $\mathrm{B}$ cell differentiation, as demonstrated by the expression of activation-induced cytidine deaminase, the enzyme required for Ig affinity maturation and class-switching, and the presence of CD138 plasma cells displaying anti-insulin reactivity. Overall, this work provides direct evidence that TLS are of critical relevance in promoting autoimmunity and chronic inflammation during autoimmune insulitis and diabetes in NOD mice. 